\title{
Success rate of repeat flexible ureteroscopy following previous failed access: An analysis of stent duration
}

Dylan T. Hoare; Timothy A. Wollin; Shubha De; Michael G. Hobart

Division of Urology, University of Alberta, Edmonton, AB, Canada

Cite as: Hoare DT, Wollin TA, De S, et al. Success rate of repeat flexible ureteroscopy following previous failed access: An analysis of stent duration. Can Urol Assoc J 2021 January 4; Epub ahead of print. http://dx.doi.org/10.5489/cuaj.7064

Published online January 4, 2021

$* * *$

\section{Abstract}

Introduction: Approximately $8 \%$ of patients that undergo therapeutic or diagnostic ureteroscopy will have the procedure aborted and ureter stented due to failed access. The primary objective of this study was to assess mean stent duration prior to repeat ureteroscopy and to calculate the associated successful access rate.

Methods: This retrospective, descriptive study evaluated all patients undergoing interval ureteroscopy following a failed procedure by endourologic surgeons at the University of Alberta from 2016-2018. Patients declining interval ureteroscopy, or those with malignant/known ureteral strictures were excluded from the study. The primary outcome measures were median time to salvage ureteroscopy and the rate of successful access of the repeat procedure.

Results: A total of 119 patients were identified as having a failed ureteroscopy during our study period. First-time and recurrent stone formers accounted for 64 (53.8\%) and 47 (39.5\%) patients, respectively. Median stent duration to second procedure was 17 days (average 20, range 10-84). Most patients had their repeat ureteroscopy at 14 days or greater $(81.5 \%)$; $22(18.5 \%)$ patients had their repeat ureteroscopy between 10 and 13 days. The success rate of a second ureteroscopy after stenting was $99.2 \%$ (118/119).

Conclusions: Ureteric stenting following failed ureteroscopy leads to exceedingly high rates of successful access at interval procedure (99.2\%). The standard duration of ureteric stenting employed at our institution is two weeks. Of the patients that underwent an accelerated second procedure (between 10-13 days of stenting), all had successful access at their interval procedure. 


\section{Introduction}

Approximately $8 \%$ of patients undergoing therapeutic or diagnostic ureteroscopy (URS) at our centre will have their procedure aborted due to an inhospitable ureter in what has been deemed "failed access."1 Difficult access may be attributed to several processes including spasm, stenosis, stricture and tortuosity. For failed access, the traditional approach at our centre has been to place a ureteric stent and proceed with an interval procedure in 2 or more weeks. Stents facilitate passive dilation, a process of gradually decreasing muscle tone and peristalsis with increased urine flow. Stents unequivocally improve the ability to access the ureter at the time of interval procedure. ${ }^{2,3}$ Currently, there is no evidence-based duration for indwelling ureteric stents after which interval or salvage ureteroscopy should be attempted. The primary objective of this study was to assess the contemporary mean stent duration prior to repeat ureteroscopy at our centre and to calculate the associated successful salvage access rate. A pre-defined secondary analysis was targeted towards patients who undergo early repeat ureteroscopy (less than 2 weeks post-URS). We hypothesized that our current practice could be shifted towards earlier interval ureteroscopy given the adverse events associated with ureteral stents (irritative voiding symptoms, flank pain, infection) and that this would not adversely impact our successful salvage

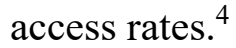

\section{Methods}

This was a retrospective, multi-site, multi-surgeon descriptive study evaluating ureteroscopy patients from January 2016 to March 2018 at the University of Alberta. Patients were identified through a billing code query within our local electronic medical record for ureteroscopy. Six hundred thirty-two patients were identified through this means. Those undergoing a secondary, interval or salvage ureteroscopy were easily identified from this cohort.

Inclusion criteria comprised all those with failed access for lithotripsy or diagnostic ureteroscopy and presumed primary failure from spasm or tortuosity. To be eligible for study inclusion, both procedures must have been performed by one of our local endourologists to minimize the confounding effect of technical ability from non-endourology trained surgeons. Patients were excluded from the study if they declined interval ureteroscopy or if the etiology of failed access was a benign or malignant stricture.

Patients meeting inclusion and exclusion criteria were queried further. Dates of primary and secondary procedures were collected, in addition to whether the procedure was diagnostic or therapeutic. When reported, the size of the stent utilized was recorded. If intracorporeal lithotripsy was performed, maximum stone size and stone location (ureteric vs renal) were documented.

The primary outcome was the duration from primary ureteroscopy to secondary URS and the rate of successful ureteric or renal access. A pre-defined secondary outcome analysis was set 
to compare those patients who underwent accelerated salvage procedures (less than 2 weeks) to those who underwent a more traditional stent duration ( 2 or more weeks).

Descriptive statistics were used for our primary and secondary outcomes. Comparison of successful access rate between accelerated and traditional stent durations was completed using Fisher's exact test in GraphPad Prism version 8.0.0 for Windows, GraphPad Software, San Diego, California USA. Statistical analysis was unblinded and no outliers were excluded. Internal Review Board approval was attained.

\section{Results}

One hundred nineteen patients met inclusion/exclusion criteria. Median age was 57 years. The median time from initial referral to primary URS was 55 days with significant variability (STD $+/-400)$. One hundred eleven $(93.3 \%)$ procedures were performed for stone disease whereas only 8 were performed for diagnostic purposes (6.7\%). For those undergoing planned lithotripsy, $50(45.1 \%)$ had stones in the ureter, $41(36.9 \%)$ within the kidney and $20(18.0 \%)$ within both the kidney and ureter. 64 (57.7\%) were first-time stone formers whereas 47 (42.3\%) were recurrent. Mean maximum stone dimension was $0.8 \mathrm{~cm}(\mathrm{STD}+/-0.4)$. Of those patients with reported stent sizes, the most common variety was a $6 \mathrm{Fr}$ by $26 \mathrm{~cm} \mathrm{JJ}(52 / 79-65.8 \%)$. Other varieties included 6 Fr by $24 \mathrm{~cm}(13 / 79-16.5 \%), 6$ Fr by $28 \mathrm{~cm}(3 / 79-3.8 \%), 4.8$ Fr by $26 \mathrm{~cm}$ $(3 / 79-3.8 \%), 7$ Fr by $26 \mathrm{~cm}(7 / 79-8.9 \%)$, and an antegrade 8 Fr by $22 \mathrm{~cm}(1 / 79-1.3 \%)$.

There was a wide distribution of stent durations within our study cohort, ranging from 10 to 84 days between initial failed access and subsequent salvage procedure (Figure 1). Median and mean time to interval ureteroscopy were 17 and 21 days respectively. No patients had an interval procedure in less than 10 days. Nineteen (16.0\%) patients underwent an accelerated salvage ureteroscopy in the 10-13 days period (Table 1). Seventy (58.8\%) patients underwent repeat procedure between 14 and 21 days while another $30(25.2 \%)$ patients were outside of this interval. Overall secondary ureteric/renal access rate was 99.2\% (118/119). One patient had failed access at their interval procedure that took place at a 14-day interval. Persistent ureteric spasm was the reported etiology of failed access. A stent was reinserted and a third ureteroscopy was successfully completed 14 days later. Comparing the accelerated cohort (10-13 days) to a traditional stent duration ( $2+$ weeks), there was no significant difference in the successful secondary access rate (two-tailed $\mathrm{p}=1.0$ ).

\section{Discussion}

Ureteric stents hold an undisputed role in the realm of endourology, serving to decompress infected and obstructed collecting systems, mitigating iatrogenic injuries, and specific to this study, facilitating passive ureteric dilation following failed initial access. For over a century, we have known of the merits and luminal dilating capabilities of stents. ${ }^{5-7}$ Despite decades of routine use, the nuances of stent placement remain to be fully defined. A recent Cochrane meta-analysis evaluating the use of stents following ureteroscopy highlighted the significant uncertainty and 
heterogeneity in this field. ${ }^{8}$ Most outcome measures were of low or very low evidence, with no consensus on whether ureteric stents improve unplanned emergency departments visit rates, secondary interventions, narcotic requirements, urinary tract infections or ureteral strictures. This uncertainty extends into the realm of pre-stenting and failed access.

Failed access is a relatively common occurrence. In an internal review, we determined our failed access rate to be $8 \%$ of all ureteroscopy patients. ${ }^{1}$ When access fails, the default decision is to place a ureteric stent and proceed with an interval or salvage secondary procedure. Most of the evidence on this is extrapolated from studies on the use of pre-stenting. ${ }^{3,9-10}$ There is a significant dearth of literature pertaining directly to the scenario of interest. And despite the increasing acceptance of balloon dilation, stenting and returning for a secondary URS maintains a significant safety margin, particularly for the general urologist, and is deserving of greater investigation. $^{11,12}$

One prospective cohort study from China evaluated 59 patients in this salvage setting, comparing the use of double-J ureteric stents to ureteral catheters. ${ }^{13}$ Double-J stents were placed if a guide wire could be successfully placed. These patients proceeded to interval ureteroscopy after 2 or more weeks, $97.5 \%$ of whom had successful secondary access. Ureteral catheters affixed to a Foley catheter were left if stone impaction prevented the passage of a guide wire on initial ureteroscopy. These patients proceeded to repeat URS in 3 to 5 days. Like the double-J cohort, secondary access was highly successful (100\%) following placement of the open-ended catheter. Although an open-ended catheter may not be a practical modality for managing failed access, the success of the procedure and the short indwelling duration supports our finding of a relative equivalence with accelerated stent duration.

Although the prevalence of stent-associated morbidity varies throughout the literature, there is an irrefutable ability for stents to impact quality of life. Joshi reported that stents may reduce quality of life in excess of up to $80 \%$ of patients. ${ }^{4}$ Great efforts have been made to reduce these adverse effects, including pharmacologic intervention, selective utilization and early removal. ${ }^{14}$ There is optimism that moving forward, engineering innovations will mitigate some of this stent-associated morbidity. Material composition, diameter, length, shape, biodegradability, coating and drug-elution are all stent properties undergoing continued investigation. ${ }^{15,16}$ The delicate balance of cost and benefit, in conjunction with meaningful industry investment remains to be achieved, however. As this field evolves, the desire to limit stent duration may wane. For now, limitation of stent duration ranks among the more practical approaches to decreasing morbidity.

Accelerated stent duration has the potential to offer more than simply reduced stent morbidity. Tertiary care centres, such as our facilities in Edmonton, Alberta, service significant geographic areas. Patients travelling from rural and remote areas are often subsidized by the government to travel and undergo URS. In addition, extended leaves of absence are required for these patients undergoing repeat procedures away from home. In Edmonton, our operative 
theatres are also subject to slow-downs and shut-downs around seasonal events. These factors accounted for the patients with significantly extended stent durations ( $\geq 1$ month). If failed access procedures can be offered at an earlier interval, increased flexibility in surgical bookings and reduced leave from home and work may be achieved. In our current cohort, if all patients were booked for a repeat procedure in ten days, there would be a 51\% reduction (absolute reduction of 1245) in total stented days (1190 stented days versus 2435 stented days). Although we did not perform a cost-benefit analysis, it is our projection that this reduced stent duration would yield a significant reduction in morbidity and expected cost savings due to less time off work.

Both the retrospective design of this study and the small sample size have proven to be two of the greatest limitations in our findings. We believe the limited sample size reflects the low incidence of failed ureteric access at our centre. Given the retrospective nature of the study, attempts were made to limit confounding variables, such as surgeon experience with endourology. We accomplished this by limiting data analysis to only those surgeons with endourology subspecialization. In doing so however, the generalizability of our results may have been impaired. It is conceivable that general urologists could be less aggressive with various ureteric maneuvers, thereby increasing rates of failure from those observed in our study.

\section{Conclusions}

Ureteric stenting following failed ureteroscopy leads to exceedingly high rates of successful access at interval procedure $(99.2 \%)$. The standard duration of ureteric stenting employed at our institution is two weeks. Of the patients that underwent an accelerated second procedure (between 10-13 days of stenting), all had successful access at their interval procedure. This suggests that in a resource limited system, when considering options to reduce stent-related morbidity, accelerated time to salvage URS may be considered. Despite the limitations of our study, these findings warrant further prospective evaluation. 


\section{References}

1. Lavoie CA, Levine M, Schuler, TD et al. Understanding failures in getting it up: The prevalence and predictors of failed ureteral access in ureteroscopy. Can Urol Assoc $J$ 2020;15. Epub 2020 Aug 7.

2. Chu L, Sternberg KM, Averch TD. Preoperative stenting decreases operative time and reoperative rates of ureteroscopy. $J$ Endourol 2011;25:751-4.

3. Shields JM, Bird VG, Graves R et al.. Impact of preoperative ureteral stenting on outcome of ureteroscopic treatment for urinary lithiasis. J Urol 2009;182:2768-74.

4. Joshi HB, Stainthorpe RP, MacDonagh FX et al. Indwelling ureteral stents: Evaluation of symptoms, quality of life and utility. J Urol 2003;169:1065-9.

5. Wiseman JL. Observations on the stimulating influence of temporary rubber splinting on regeneration following ureteral resection: An experimental study. Br J Urol 1934;6:1126.

6. Shoemaker GE. An improvement in the technique of catheterization of the ureter in the female. Ann Surg 1895;22:650.

7. Hubert KC, Jeffrey SP. Passive dilation by ureteral stenting before ureteroscopy: Eliminating the need for active dilation. J Urol 2005;174:1079-80.

8. Ordonez M, Hwang EC, Borofsky $M$ et al. Ureteral stent versus no ureteral stent for ureteroscopy in the management of renal and ureteral calculi. Cochrane Database Syst Rev 2019;2

9. Netsch $\mathrm{C}$, Knipper S, Bach $\mathrm{T}$ et al. Impact of preoperative ureteral stenting on stone-free rates of ureteroscopy for nephroureterolithiasis: A matched-paired analysis of 286 patients." Urology 2012;80:1214-20.

10. Rubenstein RA, Zhao LC, Loeb S et al. Prestenting improves ureteroscopic stone-free rates. J Endourol 2007;21:1277-80.

11. Bourdoumis A, Tanabalan C, Goyal A et al. The difficult ureter: Stent and come back or balloon dilate and proceed with ureteroscopy? What does the evidence say? Urology 2014;83:1-3.

12. Ordon M, Andonian S, Blew B et al. CUA Guideline: Management of Ureteral Calculi. Can Urol Assoc J 2015;9:E837.

13. Ji C, Gan W, Guo H et al. A prospective trial on ureteral stenting combined with secondary ureteroscopy after an initial failed procedure. Urol Res 2012;40:593-8.

14. Chew BH, Seitz C. Impact of ureteral stenting in ureteroscopy. Curr Opin Urol 2016;26:76-80.

15. Sali GM, Joshi HB. Ureteric stents: Overview of current clinical applications and economic implications. Int J Urol 2020;27:7-15.

16. Lundeen CJ, Forbes CM, Wong VKF et al. Ureteral stents: The good, the bad and the ugly. Curr Opin Urol 2020;30:166-70. 


\section{Figures and Tables}

Fig. 1. Distribution of indwelling stent durations following failed primary ureteroscopy to interval procedure.

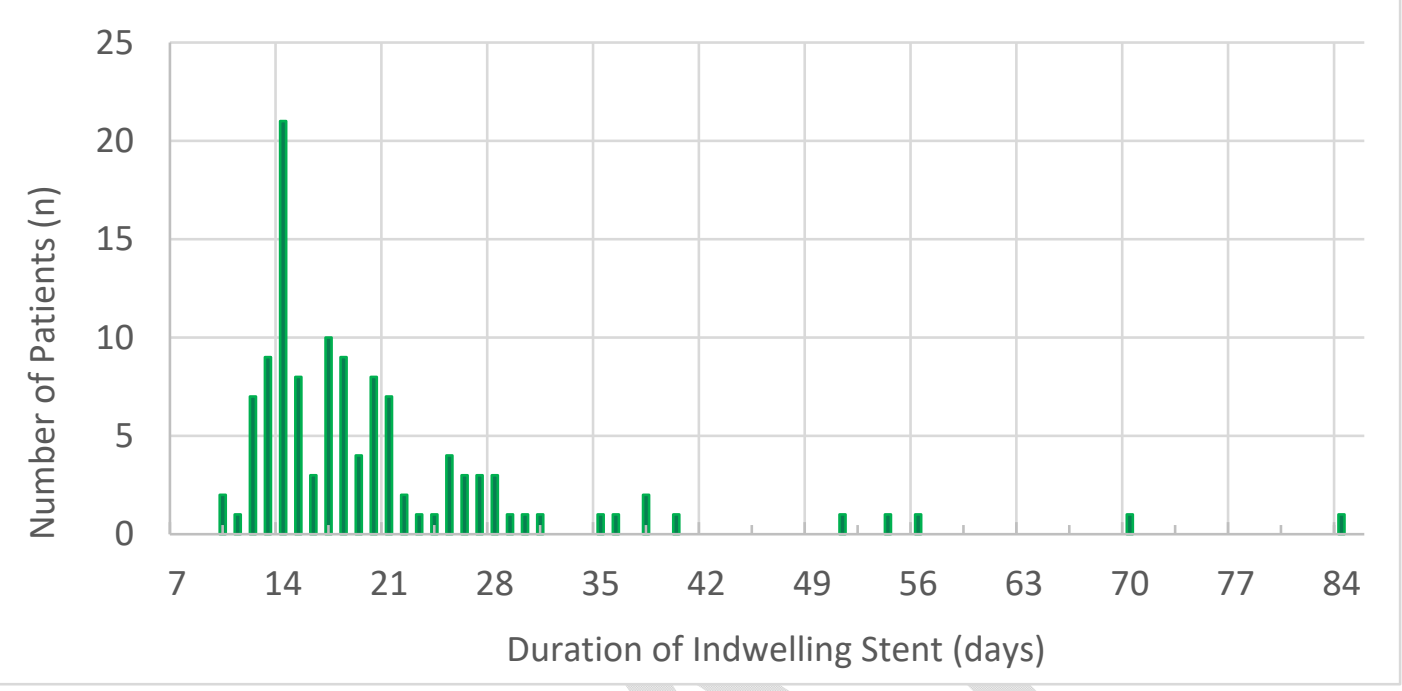

\begin{tabular}{|c|c|c|}
\hline & $\begin{array}{c}\text { Normal stent duration } \\
(\geq 14 \text { days })\end{array}$ & $\begin{array}{c}\text { Accelerated stent duration } \\
(<14 \text { days })\end{array}$ \\
\hline Patients (n) & 100 & 19 \\
\hline Mean age (years, SD) & $55.7( \pm 14.7)$ & $56.8( \pm 7.8)$ \\
\hline Stent duration range (days) & $14-84$ & $10-13$ \\
\hline Ureteroscopy indication $(\mathrm{n}, \%)$ & +2 & \\
\hline Nephrolithiasis & $93(93.0 \%)$ & $18(94.7 \%)$ \\
\hline Diagnostic & $7(7.0 \%)$ & $1(5.3 \%)$ \\
\hline \multicolumn{3}{|l|}{ Stone location $(\mathrm{n}, \%)$} \\
\hline Ureter & $42(45.2 \%)$ & $8(44.4 \%)$ \\
\hline Kidney & $34(36.5 \%)$ & $7(38.9 \%)$ \\
\hline 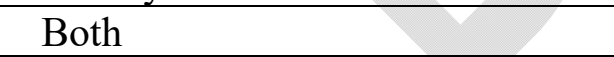 & $17(18.3 \%)$ & $3(16.7 \%)$ \\
\hline $\begin{array}{l}\text { Mean maximum stone size } \\
(\mathrm{cm}, \mathrm{SD})\end{array}$ & $0.8( \pm 0.4)$ & $0.9( \pm 0.4)$ \\
\hline Successful secondary access (n, \%) & $99(99 \%)$ & $19(100 \%)$ \\
\hline
\end{tabular}

SD: standard deviation. 